\title{
Editorial: finite geometries
}

\author{
Dina Ghinelli ${ }^{1}$ - Dieter Jungnickel ${ }^{2}$. \\ Michel Lavrauw ${ }^{3}$. Alexander Pott ${ }^{4}$
}

Finite geometry is a fascinating area in mathematics at the crossroads of combinatorics, algebra and geometry. The subject embraces a wide range of problems related to affine and projective spaces over finite fields, and has many connections to current research topics in coding theory and cryptography. This volume contains 15 papers addressing typical problems in the area, in particular: problems on codes, curves, arcs, hyperovals, graphs, nets, projective planes, inversive planes, and polar spaces.

Nakić and Storme develop geometric tools to investigate the extendability of particular classes of constant dimension random network codes.

Coşgun, Özbudak and Saygı obtain new results for the number of rational points of a class of Artin-Schreier type algebraic curves, both in odd and even characteristic.

Korchmáros and Nagy study three-nets which can be coordinatized by a dissociative loop $G$ but not by a group in a projective plane over algebraically closed fields of finite characteristic, obtaining structural results on $G$.

Beelen and Piñero investigate the duals of Grassmann codes as well as the affine Grassmann codes, classifying their minimum weight codewords.

De Winter, Kamischke and Wang use results from graph theory to develop new techniques to study regular partial difference sets in Abelian groups.

Bartoli, Giulietti and Zini give a construction of complete $(k, 3)$-arcs in finite projective planes from quartic curves using the theory function fields.

De Bruyn studies sufficient conditions for a hyperplane of the five-dimensional Hermitian dual polar space to arise from the Grassmann embedding.

Rousseva, Landjev and Storme investigate the extendability of arcs associated with Griesmer codes, introducing the notion of $t$-quasidivisible arcs in a finite projective space.

\footnotetext{
Michel Lavrauw

michel.lavrauw@unipd.it

1 Rome, Italy

2 Augsburg, Germany

3 Padua, Italy

4 Magdeburg, Germany
} 
Gillespie, Giudici, Hawtin and Praeger consider subsets of the vertex set of a Hamming graph and classify two-neighbour transitive codes, with minimum distance $\geq 5$, which allow a faithful action of their automorphism group on the set of entries of the Hamming graph.

Kusejko applies combinatorial properties of pencils of conics in a projective plane to investigate the existence of collineations which maps two given conics into two conics in diagonal form.

Betten classifies packings of PG(3,3), i.e., sets of pairwise disjoint spreads partitioning the set of lines of PG $(3,3)$.

Cossidente, Marino and Pavese construct families of non-linear maximum rank distance codes and optimal constant rank codes using the geometry of Segre varieties and Veronese varieties.

Hui studies inversive planes by means of an incidence structure induced by one of its circles, using the Bundle Theorem, which gives a sufficient condition for four points to be concircular.

Cooper and Penttila complete the classification of transitive hyperovals in projective planes with groups of order divisible by four.

Fancsali and Sziklai investigate collections of subspaces of a projective space defined in terms of their intersection with other subspaces. 